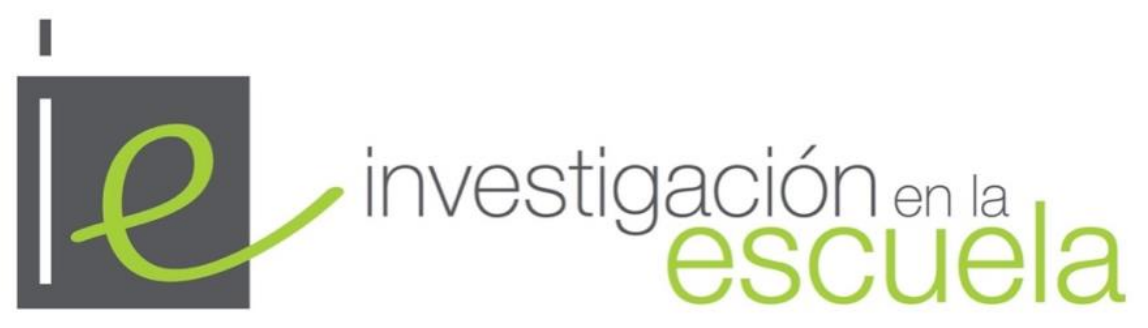

Revista de Investigación e Innovación Educativa nº 102, 2020 | e-ISSN 2443-9991

\title{
Tejiendo sentidos y aprendizajes en la formación inicial con la Lesson Study. Un estudio de casos
}

Weaving senses and learning in the initial formation with the Lesson Study. A case study

iD Dra. Cristina Rodríguez Robles es Becaria de Investigación en la Facultad de Educación de la Universidad de Málaga (España)·crobles@uma.es·https://orcid.org/0000-0002-4899-5081

iD Dra. Encarna Soto Gómez es Profesora Titular en la Facultad de Educación de la Universidad de Málaga (España)·esoto@uma.es·https://orcid.org/0000-0001-5758-1684

Cómo citar este artículo

Rodríguez Robles, C. y Soto Gómez, E. (2020). Tejiendo sentidos y aprendizajes en la formación inicial con la Lesson Study. Un estudio de casos. Investigación en la Escuela, 102, 109-121. doi: http://doi.org/10.12795/IE.2020.1102.08

Resumen. La formación inicial requiere una transformación profunda de los procesos de enseñanza y aprendizaje para estimular la reconstrucción del conocimiento práctico de los futuros docentes. La Lesson Study (LS), como numerosas investigaciones evidencian, puede convertirse en un privilegiado escenario de reflexión y acción cooperativa para estimular el desarrollo del pensamiento práctico de los docentes en activo. En el ámbito de la formación inicial del profesorado, aunque con menos estudios y evidencias, este modelo de investigación alumbra también interesantes hallazgos. En el presente artículo presentamos los resultados de un estudio de caso sobre una experiencia que incorpora la LS a través del Prácticum y el Trabajo Fin de Grado en la formación inicial. Los principales hallazgos de esta investigación señalan que la LS, a través de sus diferentes fases, es el factor clave para la reconstrucción del conocimiento práctico del caso investigado. En concreto, el énfasis en centrar la mirada en los niños y niñas, el diseño y desarrollo cooperativo, reflexivo y tutorizado de la propuesta didáctica, aparecen como las influencias más significativas.

Abstract. Initial training requires a profound transformation of the teaching and learning processes if we want to stimulate the reconstruction of the practical knowledge of future teachers. The Lesson Study (LS), as numerous investigations show, can become a privileged setting for reflection and cooperative action to simulate the practical thinking of active teachers. In the field of initial teacher training, although with fewer studies and evidence, this research model also sheds light on interesting findings. In this article we present the results of a case study on an experience that incorporates LS into initial teacher training through the Practicum and Final Degree Project. The main findings of this study point to the LS, through its different phases, as key factor for the reconstruction of the practical knowledge of investigated case. Specifically, the emphasis on focusing on children, cooperation, the continuous relationship between theory and practice, and tutoring as facilitation appear as the most significant influences.

\section{Palabras clave $\cdot$ Keywords}

Lesson Study, prácticum, conocimiento práctico, tutorización, formación inicial docentes, cooperación, reflexión. Lesson Study, practicum, practical thinking, tutoring, initial training education, cooperation, reflection. 


\section{Introducción}

La imprenta, ahora abolida, ha sido de los peores males del hombre, ya que tendió a multiplicar hasta el vértigo textos innecesarios. (Borges, 2005, p. 110).

El exceso de difusión de la imprenta, internet lo desbordó inaugurando la denominada era de la información, o desinformación, donde el reto educativo ya no reside en memorizar contenidos, sino en saber detectar y aplicar críticamente el saber para orientar e interpretar los modos de intervenir en la realidad. $\mathrm{Al}$ igual que el doctor Frankeinstein creía que cosiendo partes de humanos grandiosos, pero muertos, podría crear un ser humano perfecto, nuestro sistema de formación inicial pretende que los futuros docentes cosan y revivan los contenidos incluidos en un currículum impersonal, descuartizado, jerarquizado y muerto cuando lleguen a la práctica (Pérez Gómez, 2012).

Aunque las Competencias del Grado de Educación Infantil (ORDEN ECI/3854/2007 de 27 de diciembre) plantean que los futuros docentes deben aprender a ayudar al alumnado a construir sus propios aprendizajes, generando currículos integrados que fomenten el desarrollo colectivo y personal en contextos diversos, la concreción de los planes de estudios después de Bolonia seguían promoviendo programas formativos atomizados, con un fuerte sesgo teórico y un prácticum situado, generalmente, al final del periodo de formación, como contexto de aplicación más que de reflexión práctica.

Reconocer el prácticum como un proceso importante, aunque incierto, para la formación inicial de los docentes, porque el alumnado tiende a repetir su experiencia escolar, no es un descubrimiento actual (Blanco,1999; Pérez Gómez, 1999). Como señalan Zeichner \& Gore (1990), podemos definir y entender al profesorado como prisionero del pasado (experiencias previas) y del presente (presiones del contexto), con escasa oportunidad para reconstruir un conocimiento práctico primitivo apenas hilvanado con referentes teóricos del periodo de formación. Tal y como señalan Cladinin \& Husu (2017), no se trata solo de dar respuestas, soluciones o teorías claras, sino de comprender las múltiples perspectivas que intervienen en cómo estamos pensando y participando en las prácticas y políticas de la educación del profesorado y, sobre todo, de cómo establecer una relación dialéctica entre la investigación y la práctica de la formación docente.

\section{Aprendizajes reversibles, conscientes e inconscientes}

Cuida tus pensamientos, porque se convertirán en tus palabras. Cuida tus palabras, porque se convertirán en tus actos. Cuida tus actos, porque se convertirán en tus hábitos. Cuida tus hábitos, porque se convertirán en tu destino. (Gandhi).

Investigaciones en neurociencia (Damasio, 2003; Blakemore y Frith, 2005; Bueno i Torrens, 2017) nos ayudan a comprender, cuidar y retar las palabras, actos y hábitos del futuro profesorado. Los instintos de supervivencia básicos, que consideran lo conocido bueno y lo desconocido peligroso, son influyentes notables en las decisiones docentes. Pero si además añadimos que cuanto más se repita una respuesta más posibilidades tiene de que se reproduzca de forma automática, comprendemos que tras años de obediencia, repetición y culto a la teoría, es difícil construir una cultura educativa creativa que dialogue con la realidad.

El cerebro procesa y almacena sus aprendizajes, de forma necesaria, en dos dimensiones diferenciadas: el consciente donde se desarrollan nuestras teorías declaradas, la verbalización de lo que hacemos o sabemos; y el inconsciente donde se desarrollan nuestras teorías en uso (Argyris, 1993; Khaneman, 2015), aquellas que se construyen a través de hábitos y/o experiencias emocionales que nos impactan a lo largo de nuestra vida, denominado conocimiento práctico (Pérez Gómez, 2012).

Sin embargo, al estar conectadas ambas dimensiones del cerebro nuestro consciente puede observar las respuestas automáticas inconscientes, es decir nuestras teorías en uso, y analizarlas en relación a los saberes acumulados: nuestras teorías proclamadas. Este proceso permite elaborar nuevas respuestas meditadas, capaces de influir e incluso inhibir respuestas primitivas inconscientes, creando otras respuestas automáticas informadas, lo que Pérez Gómez (2012) denomina pensamiento práctico. Un pensamiento constituido por todos los recursos (conscientes e inconscientes) que utilizamos los seres humanos cuando intentamos comprender, diseñar e intervenir en una situación concreta de la vida personal o profesional, pero tamizadas por un proceso reflexivo continuo y sistemático que, indefinidamente, cuestiona la virtualidad educativa del conocimiento práctico.

El cultivo de la teoría no implica necesariamente la transformación de la práctica. En cambio la práctica reflexiva permite abrir un flujo de reconstrucción y relación entre la teoría y la práctica (Alsina y Batllori, 
2015; Martin \& Clerc-Georgy, 2015). Es decir, convertir la teoría en verdadera herramienta de compresión e inspiración práctica y a la práctica en detonante de reflexiones teóricas.

La formación inicial requiere con urgencia una compleja transformación (Grossman et al., 2009; Darling-Hammond \& Oakes, 2019). Necesita ofrecer oportunidades para generar hábitos reflexivos informados en contextos de investigación sobre la práctica y la teoría, basados en un diálogo recíproco y nutrido entre el consciente y el inconsciente, para estimular la necesaria y continua reconstrucción del conocimiento práctico en pensamiento práctico. Ahora bien, cabría preguntarse: ¿qué procesos y estrategias formativas estimulan la emergencia del conocimiento práctico y la construcción del pensamiento práctico?, ¿ayudan las Lesson Study (LS) a desvelar y en su caso reconstruir los componentes más implícitos del conocimiento en acción de los docentes?

\section{Principios pedagógicos que inspiran la innovación educativa en la formación inicial}

A nuestro entender, existen tres principios básicos que debemos incorporar en la formación inicial de docentes que han de funcionar como sistema (Soto et al., 2019), es decir, en estrecha relación, si queremos promover la necesaria reconstrucción del conocimiento práctico: la reflexión vinculada a la práctica, la cooperación y la tutorización.

En relación con la reflexión, como formadores de docentes necesitamos generar contextos educativos donde los futuros docentes se conviertan en investigadores de su propias rutinas y concepciones inconscientes, es decir, donde reflexionen sobre la teoría en relación con su práctica y su conocimiento práctico y se eduquen a través de su propia investigación, (Elliot, 1999; 2015). Siguiendo a Pérez Gómez et al. (2015), existen dos movimientos que componen este necesario diálogo entre teoría y práctica: la teorización de la práctica (Hagger \& Hazel, 2006), que supone la revisión y el cuestionamiento de nuestras teorías en uso a la luz de experiencias e investigaciones educativas relevantes; y la experimentación de la teoría, que implica el tránsito de la teoría a la práctica y su investigación, es decir, el proceso de traducir los cuerpos teóricos en elementos de significado para diseñar y desarrollar nuestras teorías en uso. Para que este proceso permita el desarrollo de niveles de razonamiento de orden superior y competencias transversales propias del S. XXI (Prieto y Giménez, 2020) debe ser activo, situado y sistemático, estableciendo vínculos constantes e interrogativos entre la teoría y el análisis de la propia práctica (Korthagen, 2010).

Incorporar la cooperación significa generar contextos que permitan aprender en relación, es decir, iniciar un viaje que parte del sujeto para volver a sí mismo enriquecido por el contraste y la interacción con el otro. Una oportunidad para ordenar y releer su propia experiencia en relación con los demás (Alsina \& Batllori, 2015). Fomentando la generación de espacios reflexivos, organizados y comprometidos pedagógicamente entre iguales (Lamb et al., 2012).

Por tanto, esta cooperación debe implicar la tutorización, como parte del proceso reflexivo que acompaña, contrasta y cuestiona. Numerosos estudios han demostrado que la vaga planificación y seguimiento de las experiencias prácticas pueden obstaculizar el aprendizaje de los estudiantes de magisterio (Pérez Gómez, 1999et al., 2020). En la investigación desarrollada por Pérez Gómez (1999) acerca de las influencias del periodo de prácticas sobre el pensamiento, los intereses, actitudes y comportamientos pedagógicos de los futuros docentes en las Facultades de Educación de Andalucía, encontraron dos procesos simultáneos que pueden ayudarnos a interpretar esta relación, la socialización de los estudiantes durante el periodo de prácticas en una pedagogía vulgar, reproductiva más autoritaria, impersonal y burocrática, junto con una ausencia total de una tutorización que estimule el distanciamiento crítico y la mediación reflexiva necesaria que permitan compensar y contrastar los poderosos influjos socializadores del medio escolar. Un tránsito incómodo que hay que recorrer acompañado de una tutorización que interrogue, motive, apoye y sobre todo ofrezca oportunidades e instrumentos conceptuales para analizar el valor educativo de su práctica.

\section{La experiencia: la Lesson Study, una oportunidad para recrear el conocimiento práctico}

La LS es una estrategia de formación docente cooperativa que nació hace más de un siglo en Oriente y que actualmente engloba multitud de versiones a nivel internacional (Soto et al., 2020). Ofrece un modelo sencillo que se estructura en ciclos de investigación - acción (diseño - desarrollo - observación - evaluación - mejora), con la particularidad de que todo el proceso de investigación y acción centra la mirada en el proceso de aprendizaje del alumnado. 
Por tanto, es una estrategia que permite convertir a los futuros docentes en aprendices de su alumnado (Norwich et al., 2014), así como fusionar indefinidamente las experiencias de las escuelas con las teorías, multiplicando las oportunidades de aprendizaje en la formación inicial docente y ayudando a generar habilidades prácticas (Zeichner, 2010). Así es como diferentes investigaciones evidencian que la LS mejora el conocimiento implicado en la práctica docente (Xu \& Pedder, 2015).

Sin embargo, en formación inicial y sobre todo en el contexto occidental, la LS es todavía una metodología escasamente explorada (Elliot, 2016) que afronta los siguientes retos y que constituyen el punto de partida y análisis del caso investigado:

- la dificultad temporal en los programas de prácticas para poder realizar un ciclo completo y disfrutar de tiempos relajados para la reflexión (Chassels \& Melville, 2009);

- la escasa experiencia y autonomía docente de los estudiantes en los contextos de prácticas, que impiden la emergencia de sus propios objetivos y diseños de enseñanza (Po Yuk, 2011);

- la dedicación que supone la tutorización de la LS (Po Yuk, 2011), así como, la dificultad de integrarla en los programas de formación y organización del prácticum en las escuelas para propiciar la observación mutua (Soto et al., 2020); (Chassels \& Melville, 2009).

\section{Diseño metodológico}

\subsection{Foco de investigación}

El objetivo fundamental de esta investigación es detectar las potencialidades pedagógicas de la LS para la reconstrucción del conocimiento práctico de estudiantes de educación, a través de un estudio de caso sobre la experiencia desarrollada en el Trabajo Fin de Grado (TFG) y Prácticum III del cuarto curso del Grado de Educación de Infantil de la Facultad de Ciencias de la Educación de la Universidad de Málaga.

\subsection{Método y procedimientos de investigación}

Para adentrarnos en un terreno tan complejo, cambiante e inacabado como el conocimiento práctico hemos optado por una investigación de enfoque cualitativo, a través de un estudio de caso. La investigación se centra en una alumna, María, que forma parte de un grupo de cinco estudiantes que van a desarrollar una LS.

Seleccionamos este grupo porque su Tutora Académica (TA) es una de las promotoras del proceso de LS en la Universidad de Málaga y ha tutorizado a este grupo durante los dos prácticums anteriores, siendo este el tercer momento de tutorización, atendiendo a la relevancia que Chassels \& Melville (2009) conceden a que la TA tenga una relación de confianza con las estudiantes, que le permita realizar un seguimiento íntimo de sus procesos y que sea especialista en LS (Po Yuk, 2011). La elección de María tuvo que ver con la facilidad de acceso a su centro de prácticas, ya que este colabora habitualmente con la universidad.

La investigación partió de la observación del conocimiento práctico de María (conocimientos, habilidades, actitudes, emociones y valores) a lo largo de todo el proceso de la Lesson Study, tanto a través de su discurso como de su práctica en el aula, en torno a tres ejes fundamentales: cómo María definía su idea de niñez, la imagen de docente y de enseñanza.

En el resultado de estos análisis encontramos como categorías emergentes: el valor de la cooperación, la tutorización y la focalización en la infancia, elementos claves para la reconstrucción de su conocimiento práctico. El programa de análisis cualitativo NVIVO nos permitió visualizar y analizar la iteración no lineal de estos seis códigos (unidades de contenido de dicha investigación) y desarrollar una compleja triangulación de los datos (Gil Flores y Perera Rodríguez, 2001).

\subsection{Tiempos, objetos e instrumentos de investigación}

En este estudio hemos observado, documentado y entrevistado a la estudiante durante todo un curso académico (octubre a junio). Empleando los siguientes instrumentos, objetos y tiempos de investigación (Tabla 1). 
Tabla 1

Instrumentos, objetos y tiempos de investigación

\begin{tabular}{|c|c|c|}
\hline Instrumentos & Objetos & Tiempos \\
\hline Observaciones & $\begin{aligned} & 6 \text { seminarios del Practicum III } \\
&- \text { Seminario presentación (S1) } \\
&- \text { Seminario Fase } 1 \text { (S2) } \\
&- \text { Seminario Fase } 2 \text { (S3 y S4) } \\
&- \text { Seminario Fase } 4 \text { (S5) } \\
&- \text { Seminario Fase } 6 \text { (S6) } \\
& 3 \text { reuniones de estudiantes durante Fase } \\
& 2 \text { (R1, R2 y R3) } \\
& 4 \text { jornadas en su centro de prácticas } \\
&-\quad 2 \text { antes de la Fase } 3 \text { (J1 y J2) } \\
&-\quad 2 \text { durante la Fase } 3 \text { (13 y J4) }\end{aligned}$ & $\begin{array}{l}\text { (9 horas de vídeo. y andio) } \\
\text { ( } 4 \text { horas de audio) } \\
\text { (21 horas de vídeo) }\end{array}$ \\
\hline Diario de campo e investigación & & 15 octubre a 30 junio \\
\hline Análisis documental & $\begin{array}{l}\text { Portafolio virtual del: } \\
\quad-\quad \text { Practicum I (PI) } \\
\quad-\quad \text { Practicum II (PII) } \\
-\quad \text { Practicum III (PIII) } \\
\quad \text { - } \quad \text { Foro de discusión (F) } \\
\text { Trabajo Fin de Grado (TFG) } \\
\text { Proyecto de Intervención Autónoma } \\
\text { (PIA) } \\
\text { Póster científico } \\
\text { Guía del Docente }\end{array}$ & $\begin{array}{l}(15 \text { sesiones }) \\
(20 \text { sesiones }) \\
(80 \text { sesiones })\end{array}$ \\
\hline Entrevistas personales & $\begin{array}{l}4 \text { semiestructuradas } \\
\quad-\quad 2 \text { antes de la Fase } 3 \text { (E1 y E2) } \\
-\quad 2 \text { tras la Fase } 3 \text { (E3 y E4) } \\
\text { Diversas charlas informales (CI) } \\
1 \text { entrevista final (EF) }\end{array}$ & ( 3 horas de audio) \\
\hline
\end{tabular}

Los extractos textuales que se recogerán en el apartado de resultados se referenciarán conforme a las siglas de los objetos de investigación, tal como se recogen en la Tabla 1.

\subsection{Contexto de investigación}

Para facilitar la comprensión del proceso, describiremos el contexto y desarrollo de la experiencia investigada, estructurada en torno a las fases de la LS. Cada fase se inaugura con un seminario, donde la TA ejerce de guía metodológica de la LS y trata de generar un ambiente de confianza para compartir inquietudes e introducir a las alumnas en hábitos de reflexión entre la teoría y la práctica. Inicialmente analizamos los portafolios del Prácticum I, II de María, así como las sesiones iniciales de prácticas, para recoger evidencias que permitieran describir su conocimiento práctico antes del proceso de LS.

Fase 1. Diagnóstico e identificación de objetivos

Las alumnas exploran y analizan sus contextos de prácticas en sus diarios digitales. Una fase de diagnóstico que pone en relación con su TA, para buscar y describir focos de reflexión en sus centros de prácticas, que den forma a los objetivos de grupo. Objetivos alimentados, a su vez, por los intereses personales de investigación, que orientarán su TFG. Según la normativa de esta Facultad, el TFG comienza tras el Practicum III (segundo semestre), pero en este caso se adelanta para generar un marco teórico contrastado que cultive el diseño grupal.

Fase 2. Diseño de la Lección Experimental (LE) y Estudio de la lección (EL)

Cooperativamente las alumnas realizan un diseño didáctico, que se concreta en un documento que el marco normativo de esta universidad denomina PIA y que en nuestro caso es la LE. Una propuesta didáctica que debe estar pensada desde la mirada de la infancia. Al mismo tiempo han de diseñar cómo van a estudiar dicha propuesta y cómo esta contribuye o no al desarrollo de sus finalidades (EL). Este documento, tutorizado por la TA, es compartido con la TP antes del desarrollo de la LS.

Fase 3. Desarrollo de la LE 
En este caso, María desarrolla la propuesta como docente en su colegio de prácticas mientras una compañera la observa y recoge evidencias.

Fase 4. Análisis y mejora LE

El grupo y la TA contrastan, analizan y evalúan la LE. Poniendo en relación sus objetivos con las evidencias recogidas a través del EL y la mejoran generando LE2.

Fase 5. Desarrollo de la LE2

En este caso, su compañera ejerció de docente en su contexto de prácticas, mientras María la observaba y recogía evidencias.

Fase 6. Revisión Final y difusión

El grupo junto a la TA evalúa el conjunto de la experiencia y se inicia el momento clave del TFG, donde cada una de ellas y a partir de un análisis individual y fundamentado de la LS o PIA proponen un diseño mejorado y centrado en el foco de indagación teórica que comenzó al inicio del prácticum. El TFG se concreta en un documento que es tutorizado por la TA y se defiende ante un tribunal y grupo de compañeras mediante un póster científico.

\section{Hallazgos de la investigación y discusión de resultados}

Durante nueve meses María vive una experiencia nueva que le ha ayudado a ajustar el traje de maestra que había venido vistiendo, a veces con tiranteces, durante los años de formación. Un proceso en el que ha tenido que descoser, a veces rasgar e hilvanar y, sobre todo, añadir tejidos nuevos, que la ayuden a llevarlo con comodidad. La sinceridad, espontaneidad y facilidad con la que María se ha dejado acompañar en esta investigación han ayudado a evidenciar los diferentes ejes conceptuales que mejor describen la transformación de su conocimiento práctico y de aquellos procesos de la LS que han podido facilitarlo.

\subsection{Tejiendo una nueva idea de infancia}

En el Prácticum I María percibía y describía los procesos de enseñanza y aprendizaje desde una concepción técnica, estandarizada y convencional:

... Mientras hacían el número 1 que venía en la ficha yo iba revisando y borrando lo que habían hecho mal para que lo repitieran. Cuando terminaban me lo tenían que enseñar y después guardarlo en su sitio (...). Ha sido una experiencia muy gratificante. He de reconocer que en ocasiones me he agobiado un poco, ya que mientras le decía a un niño que cogiera bien el lápiz, otro estaba cogiendo el rotulador sin haberle dicho que lo cogiera, y otro estaba reclamándome. Pero estoy satisfecha porque considero que he sabido controlar la situación y reconducirla cuando se desviaba para que todos siguieran las instrucciones. (PI).

Esta tendencia es percibida por su TA que, a modo de espejo, recoge sus experiencias y las devuelve intentando hacer visible su conocimiento práctico.

... Una de mis funciones como tutora es provocar tu reflexión, por ello espero que mis preguntas y comentarios te ayuden a volver a visitar la experiencia situándote en otras perspectivas (...): ¿qué sentido tienen esas tareas para ellos?, ¿están vinculadas con algo real, cotidiano, con algún problema que tienen que resolver?, ¿les tiene atrapados?, ¿a todos?, ¿comentas que se respeta sus intereses?, ¿para quién se hacen las fichas?, ¿cuánto tiempo estuvieron haciendo la ficha del primer día?, ¿podrías definir el tipo de enseñanza y de aprendizaje que desarrolla esta docente? (...) 'controlar', 'reconducir', 'no se desvíen'... ¿cómo vives y viven los niños y niñas el borrado cuando sale mal? (PI).

El cuestionamiento de la tutora saca a María de su estado de confort y la hace deambular por su diario en un balanceo de dudas y preguntas, que no encontraban respuestas en su conocimiento práctico. En busca de solidez pedagógica María solicitó a su TA lecturas que le acercasen a esas nuevas concepciones de infancia, docente y escuela que compartían en los seminarios. Un punto de inflexión, que progresivamente le lleva a construir un espíritu cultivado, comprensivo, respetuoso y admirador de la cultura de infancia. Inicialmente, solo a nivel declarativo, y posteriormente, transformando plenamente su conocimiento práctico.

Durante estos tres años de prácticas he podido formarme una imagen de la infancia muy distinta a la que tenía cuando entré en la carrera, hace cuatro años. Pero esa imagen no es más que un reflejo de los niños y niñas, ellos han sido los que han provocado que cambie esa imagen, yo sólo he tenido que pararme a escuchar sus gestos, sus palabras, el lenguaje de sus ojos... Ellos me lo decían con 
gritos silenciosos, me han demostrado lo que realmente son, niños y niñas con muchas ganas de ser escuchados y de mostrarnos todas sus potencialidades y derechos. (TFG, p. 13).

\subsection{In - corporar una nueva idea de infancia genera cambios relacionados con la enseñanza y el rol docente}

Cuando ya tuve la imagen de niño me empecé a preguntar: ¿qué puedo hacer como maestra para que ese niño crezca? (EF).

Motivada y reforzada por la voz de la infancia María comenzó un viaje lento y con algunos incidentes, donde a medida que avanzaba, agudizaba su mirada crítica, aprendiendo a fundamentar y detectar incoherencias entre la práctica y la teoría. Alejándose de esa enseñanza estandarizada y unidireccional que colonizaba su conocimiento práctico.

Cada día al reflexionar sobre lo ocurrido me sorprendo pensando en la metodología por proyectos [metodología que emplea su TP] y su finalidad. La finalidad es que sean los niños los que construyan su propio conocimiento, que sean ellos los protagonistas, que creen significados e interpretaciones con la información que van investigando. Y con eso no puedo estar más de acuerdo, pero, durante este proceso se crea "homogeneización" del conocimiento, no he encontrado otra palabra para nombrarlo, en la que enseñamos a todos los niños lo mismo (...), y no todos tienen el mismo nivel, ni aprenden al mismo tiempo. (PIII).

Apreciamos cómo María descubre qué aspectos no le identifican de la escuela que vive en su aula de prácticas.

(...) Yo estaba de acuerdo con la metodología por proyectos. Porque era una cosa nueva. Pero en este Prácticum, donde a través de la LS detenidamente hemos ido desgranando cada elemento..., ya no me ha gustado. Ha habido cosas con las que no me he sentido identificada. (EF).

Además de los Proyectos de trabajo, otro hito importante de la educación infantil, como son las asambleas de aula, empieza a rasgarse y a despertar el pensamiento práctico de María.

Dos días antes del desarrollo de la LE fuimos a observarla a su colegio de prácticas y le preguntamos: Investigadora: ¿Qué te ha parecido la asamblea?

María: [suspira y mueve la cabeza de un lado a otro insinuando duda y agobio] La asamblea..., por la experiencia que yo estoy teniendo... veo que estás más tiempo pidiendo que se callen y que atiendan que hablando. Es lo que yo siento... pero todavía no me he aclarado. (E2).

Percepción que parece ser común a sus otras compañeras, ya que cuando se preguntan si deberían hacer una presentación de la LE en la asamblea deciden quitarla:

María: Es que, si quito la asamblea, ellos van a notar que hay algo extraño, porque... si todos los días se les saluda y todos los días está esa rutina... a lo mejor, dicen: ‘¿por qué no hay asamblea?'. O a lo mejor la inseguridad es mía y los niños van al ambiente y ¡Guau! [mostrando una sonrisa].

Compañera: Yo lo haría sin asamblea. (R3).

Apreciamos cómo el peso de la homogenización y la rutina impide a María ser coherente con sus observaciones, sin embargo, sólo necesitó esa frase, para liberase de la asamblea cotidiana. Tras la LS María escribe en su diario:

En el caso de mi clase, y tras una observación diaria exhaustiva, en la que yo también he adoptado el rol de maestra en alguna de ellas, he podido comprobar que [las asambleas] se habían convertido en algo aburrido y pesado para los niños. Consulté a otras maestras del colegio sobre qué pensaban de las asambleas y todas me dijeron lo mismo, que es un espacio de encuentro muy importante en el que aprenden a respetarse los unos a los otros y el turno de palabra, no se debe quitar. Pero tras la conferencia de Alfredo Hoyuelos [propiciada por la TA] terminé de aclarar mis ideas respecto al tema, ya que recordé que para que los niños compartan sus ideas no es necesario una asamblea, sólo dejarles organizar su tiempo [como hicieron en su LE] (...) y no obligar a que estén en la asamblea cuando no quieren estarlo. Yo me plantearía quitarlas, ya que no puedo (...) desatender su bienestar y necesidades. (PIII).

Detectamos cómo María identifica qué aspectos de las escuelas y de su propio conocimiento práctico no le identifican, pero ¿qué le identifica?, ¿y qué papel juega la LS en este proceso? 


\subsection{La Lesson Study un marco privilegiado para aprender a ser docente}

La LS te pone a prueba individualmente y como grupo. En cada fase te tienes que poner de acuerdo. Hay discusiones, no las hay, hay aprendizaje, no lo hay... Por eso, cada fase te pone a prueba. Y ves hasta dónde eres capaz de llegar. (EF).

Las diferentes fases de la LS han influido en la reconstrucción del pensamiento práctico de María, uno de los hallazgos de esta investigación, ya que existen escasos estudios que describan y analicen los aprendizajes de los docentes que participan en ella (Xu \& Pedder, 2015).

Fases de diagnóstico - objetivos - diseño de la LE: un espacio de resistencias entre lo propio y lo ajeno

Al compartir sus inquietudes y el análisis de sus respectivas prácticas en la fase de diagnóstico y selección del foco encuentran, para sorpresa de las estudiantes, necesidades o intereses comunes.

María: ... Cuando falta tiempo para hacer una actividad, la quitan, pero enseguida meten otra, no se les da tiempo libre a los niños para que sean autónomos, y esto se justifica con los padres, para que vean o se lleven algo que demuestre que han trabajado (...). Se le da mucha importancia al contenido. Se plantea un juego libre, pero en medio hacen una ficha del número 1: ¿por qué?, ¿para qué? (...).

TA: Entonces... ¿qué niño queremos promover en nuestra propuesta?.

María: Un niño autónomo (S2).

El grupo acuerda diseñar un contexto educativo que estimule la cooperación, la autonomía y la creatividad diseñando una LE centrada en los ambientes de aprendizajes. Esta sintonía pedagógica inicial forja la identidad de grupo, aliviando sus inquietudes e ilusionando el diseño de una propuesta basada en "sus" finalidades y valores.

Sin embargo, un mes después de este acuerdo, en plena fase de diseño de la LE, una de las estudiantes comenta:

Compañera: Pienso que nos falta lo más importante, el qué y el para qué. No creo que el fin sea solo que se autorregulen, cooperen y creen algo, falta un fin más concreto (...) porque si el fin es solo eso, para eso montamos una fiestecilla $(\mathrm{F})$.

María y el grupo debaten sobre el tema y recurren a la TA, que acompaña estas inquietudes tratando de que el grupo particularice su nivel de reconstrucción y se identifique con sus decisiones.

María: nos centramos en el aprendizaje cooperativo, autonomía... más en eso que en un contenido, como las matemáticas, el tamaño... Yo era la primera que me preguntaba: ¿qué fin tenemos en la naturaleza? (R1).

Finalmente, el grupo decide incluir objetivos específicos porque se sentían perdidas sin ellos. Es parte de la complejidad del desarrollo de una experiencia integral donde los contenidos son el qué indisoluble de cualquier proceso de aprendizaje y que a menudo se confunden con las finalidades; como plantea Pérez Gómez (2012) son marcos amplios y complejos de reflexión y de acción, donde se integran no solo los conocimientos sino también las habilidades, actitudes, valores y emociones. Ellas identificaron con una "fiestecilla" una propuesta que fomenta la autonomía, la cooperación y la creatividad en un entorno natural, objetivos claves del currículum oficial de Infantil en Andalucía y conservan la creencia de que tienen que "enseñar algo concreto", en este caso las propiedades del agua. La TA comprende la dificultad del proceso de reconstrucción, y aunque les hace reflexionar sobre la naturaleza de sus dudas, no dicta el proceso del grupo y trata de que sean ellas las que decidan y experimenten. Un pequeño paso atrás para seguir avanzando.

En este vaivén, alguna compañera del grupo empieza a definir actividades concretas sin relacionarlas con las finalidades elegidas, como por ejemplo hacer un molino de agua, alimentando así el activismo docente que María vive y cuestiona cada día en sus diarios de prácticas y que le hace estar alerta y comentar: María: Pero eso, ¿no es manipular mucho?, mejor poner tubos a un lado y a otro para que ellos hagan un circuito si quieren. Porque si una de nuestras premisas o las premisas de los ambientes es la libre manipulación, y ahora nosotros le montamos un circuito. Ahí es donde anulamos la creatividad.

Compañera: La creatividad evidentemente, la estamos cortando. (R1).

Sin embargo, días después, cuando se entera de que va a quedarse a cargo del aula con su compañera, por una ausencia de su tutora, escribe en su diario:

El fin de semana estuve como loca buscando y creando recursos y actividades que fueran acordes con mis concepciones y que a mi compañera [otra práctica] le parecieran bien. (PIII). 
Es decir, parece que María a pesar de reflexionar y cuestionar el activismo que se vive en su aula, cuando tiene la oportunidad de organizar la jornada, su primera preocupación fue buscar actividades y recursos para la clase. Pasado unos días y después de la experimentación, escribe en su diario:

Sentí que estábamos llenando en exceso la jornada con actividades, preocupándonos de que en cada hora tuvieran algo que hacer, cosa que no me gusta cuando lo veo [en el aula de prácticas] con TP, porque eso significa que estaba apartando los intereses y necesidades de los niños"(PIII).

Estas resistencias, que convivían en el pensamiento de María, se veían reforzadas por sus observaciones en el aula de prácticas, su propia experiencia escolar y, sobre todo, por las conversaciones que mantenía con sus padres (ambos docentes).

Mi padre tiene ideas muy diferentes a las mías, pero también sé que se ha criado en otra cultura. Por ejemplo, cuando yo digo que quiero que mi clase sea de otra manera, porque al final por culpa del estrés, la falta de tiempo, el agobio, esa masiva cantidad de actividades y actividades (...). Mi padre me dice "pues ¿qué te crees?, eso es ser maestra. (CI).

Establecer los objetivos y diseñar la LE, genera un espacio controvertido de avances y resistencias, fruto de la convivencia de concepciones primitivas y contemporáneas en el conocimiento práctico. Estas dos fases iniciales de la LS se convierten así en escenarios privilegiados de intercambio, contraste, reflexión y emergencia de ideas, valores y creencias que necesitan ser reconstruidas. Un constante ir y venir que nos evoca los movimientos de asimilación, acomodación y adaptación de Piaget. La asimilación, o proceso donde inicialmente conviven no sin resistencias las ideas y experiencias nuevas con los esquemas previos, y la acomodación, donde reformulamos y elaboramos estructuras nuevas como consecuencias de estas incorporaciones. Como dice Punset (2014), "la plasticidad cerebral tiene una paradoja: una vez que has aprendido algo, aunque sea sin darte cuenta, cuesta mucho desaprenderlo, porque ya has creado un camino físico, químico y eléctrico en tu cerebro" (pp. 103-104).

Ambos movimientos, la asimilación y la acomodación, son necesarios para estimular la adaptación activa del sujeto. Un proceso donde cada uno de nosotros construimos un conocimiento subjetivo y propio como respuesta a los estímulos del ambiente.

Una de las cosas que me ha enseñado este prácticum ha sido darme cuenta de las contradicciones que tenemos nosotras y que tienen las maestras. Tú vas a ver que tú eres la más innovadora del mundo que en tu clase hay libertad que en tu clase no sé qué y después te pones a analizarlo y dices: ¡no! (S6).

En convergencia con Po Yuk (2011) y Martin \& Clerc-Georgy (2015), apreciamos que la LS genera un puente claro entre la teoría y la práctica que invita a las alumnas, y en particular a María, a ir y venir relacionando constantemente sus objetivos con el diseño de su LE. Estas primeras fases de la LS favorecen un diálogo continuo y fructífero en el conocimiento práctico de María, que, como veremos en el apartado siguiente, se instalan con mayor profundidad y coherencia en la fase de desarrollo y revisión de la LS.

Fases de desarrollo, revisión y mejora: la importancia de vivenciar nuestras teorías proclamadas

Hemos podido hacer una propuesta de intervención (...), he podido ver que sí funciona y que es lo que realmente quiero. Porque he visto que ellos están realmente a gusto y felices. Ofreciéndoles la oportunidad de ser ellos mismos, de escucharlos y de organizar su tiempo (EF).

El seguimiento de sus actuaciones y reflexiones en el aula nos ha ayudado a evidenciar la importancia de la fase de desarrollo de la LE para incorporar las nuevas ideas, teorías e imágenes de infancia, enseñanza y rol docente que María ha ido construyendo.

La idea de que aprendemos con la práctica es un clásico, pero lo interesante, en este caso, es que la experiencia y la práctica en relación con un proceso reflexivo de cuestionamiento y acción cooperativa provoca un aprendizaje que se distancia de las prácticas que observan cotidianamente con sus TP, desarrollando una intervención propia crítica y singular de forma autónoma y libre que resiste las inercias del contexto social y familiar.

Las distintas fases introducen a María en un proceso de reflexión sistemático, que pone a flor de piel su pensamiento práctico, manteniéndola al acecho de informaciones y experiencias que le ayudan a comprender este nuevo y desconcertante contexto educativo. Una inquietud constante que la LS ha estimulado a lo largo de las diferentes fases: en primer lugar, a través de la observación empática de las experiencias didácticas del prácticum en la fase de diagnóstico, en segundo lugar, con el contraste continuo con sus compañeras, lecturas y charlas tutorizadas en la fase de diseño, pero, sobre todo, y en tercer lugar, durante la fase de desarrollo de la LE, donde el cambio se hizo evidente para ella. 
En sus reflexiones finales, María toma conciencia de la transformación, no solo de sus ideas, sino de su forma de estar en el aula como maestra y destaca, como ejemplo, el cambio de percepción que ha vivido en relación con la enseñanza en la educación infantil.

En la elección de los materiales fuimos muy acertadas, bueno a mí me lo ha demostrado los resultados (...). Yo encontré en los ambientes todas las cosas que echaba de menos en la metodología por proyectos (...), todas vimos que era la metodología más acorde con la idea de niño que nos estábamos formando (EF).

[La LE] me hizo reflexionar de que, sin necesidad de imponer nada, los niños alcanzaban muchos de los objetivos que nos marca el currículo. Reflejo de que otra metodología es posible (PIII).

Finalmente, nos parece necesario destacar, los tres ejes claves que han tejido la experiencia a lo largo de la LS: la cooperación, el énfasis que la LS pone en mirar desde los ojos de los niños y niñas y la tutorización.

\subsection{La cooperación}

En todo este recorrido donde María ha tenido que enfrentarse a diferentes emociones, como parte de su conocimiento práctico, nunca ha estado sola, siempre ha ido acompañada de sus compañeras, la TA y los niños y las niñas de su aula de prácticas. La cooperación, para María, ha sido una plataforma cálida para afrontar sus miedos, empoderarse de "sus" nuevas finalidades y lanzarse a experimentar aquello que realmente quería llevar al aula, los ambientes de aprendizaje.

Trabajar cooperativamente te posibilita un refuerzo continuo, no estás sola y tus ideas tampoco lo están, ni cuando decidas llevarlas a cabo (PIII).

Una compañía cálida pero compleja, que le exige justificar, debatir y consensuar sus ideas basándose en la teoría y sus propias experiencias. Un proceso plural que le lleva a reflexionar-se y reconocer-se.

... descubres cosas que no sabías que tenías o que no sabías que pensabas, se ponen en juego tantas cosas cuando trabajas en grupo. Es una oportunidad para cambiar. (EF).

Como plantean Lamb et al. (2012), los espacios de colaboración entre iguales mejoran la capacidad reflexiva y práctica de los futuros docentes:

$\mathrm{Al}$ mismo tiempo que realizamos una propuesta pedagógica, nos estamos investigando a nosotros mismos, nuestras teorías más arraigadas, nuestras concepciones, nuestras ideas más innovadoras, pero también nuestros miedos, nuestros hábitos y nuestra forma de relacionarnos, ya que todo esto lo tienes que poner encima de la mesa para poder trabajar cooperativamente. (PIII).

\subsection{Descubrir-se en la infancia}

Recuperando a Norwich et al. (2014), el mirar desde los ojos de la infancia ha convertido el proceso vivido por María en una verdadera experiencia de aprendizaje.

Yo cambié la idea de infancia porque fueron los niños los que me dijeron que debía de cambiar. Todo lo que he aprendido ha sido gracias a los niños. En el primer Prácticum yo vi que los niños no estaban a gusto y (...) tenía que cambiar esa imagen hacia ellos. (EF).

De este modo, el prácticum, en relación con la LS, se convierte en un observatorio privilegiado para descubrir la infancia y su relación con la escuela. Cada propuesta pedagógica la analiza desde la mirada de los niños y niñas y busca su lugar como maestra en este triángulo de sentido. Gracias a la LS María ha podido observar que los niños y niñas cooperan y crean de forma autónoma si se le ofrece el contexto adecuado.

Yo tenía muchas ideas, muchas concepciones... y en Loris Malaguzzi encontré un marco en el que podía amparar esas ideas y ahí es donde empecé a crear la imagen de niño, una imagen potente, capaz... pero no podía verla en el aula como yo quería verla. (EF).

\subsection{Y, por último, la tutorización}

Algunas de las claves que mejor han definido el proceso de tutorización ha sido la confianza y la experiencia de la TA en el desarrollo de la LS.

La confianza y cercanía establecida entre María y la TA le ha ayudado a sostener y resistir emocionalmente las inercias culturales, sociales, familiares (ella es la tercera generación de profesores en su familia), que forman parte de su conocimiento práctico. Incluso en esos itinerarios controvertidos y contaminados de afectos y emociones, la tutora, con delicadeza y apertura, enfrentaba a María con aquellas creencias más arraigadas que contradecían algunas de sus aspiraciones como maestra. Una forma de 
acompañar y provocar que, lejos de ofrecer verdades, da la palabra a las estudiantes e incita a reflexionar sacándolas de su zona de confort.

Yo es que siempre que voy con dudas salgo con más dudas. Porque claro ella [TA] no nos quiere

dar la respuesta o decir lo que es adecuado, porque no hay nada correcto" (R2).

La experiencia de esta tutora en relación a las LS, tal y como señala Po Yuk (2011), le ha permitido prever y comprender los procesos y tiempos de aprendizaje que van a vivir las alumnas en cada una de las fases de la LS.

TA: Cuando vamos a diseñar emerge lo más profundo de nosotras, nuestro inconsciente, así que tenemos que definir bien nuestros objetivos (S2).

Para ello, cuidó y diseñó de forma detallada cómo integrar y guiar una LS con dos ciclos en la formación inicial, estableciendo unos tiempos claros y relajados de reflexión y acción, donde el grupo de estudiantes pudiera sentirse libre para diseñar su propia práctica y generar un contraste reflexivo permanente durante los seminarios, el diario y las tutorías individuales. Un ecosistema complejo donde María ha podido expresar y analizar sus vivencias durante el prácticum, así como sus repercusiones en su conocimiento práctico.

\section{Conclusiones}

María sitúa a la LS como el factor clave para la reconstrucción de su conocimiento práctico. La LS con sus diferentes fases le ha permitido simultanear el desarrollo del marco teórico, el diseño de la propuesta y el desarrollo y análisis de su práctica. Este complejo proceso, favoreció el pensamiento práctico de María creando conexiones constantes entre la teoría y su práctica, percibiendo el carácter holístico del aula.

Que los propósitos nazcan de un diagnóstico personal y cooperativo, le permitió detectar la homogenización que se vive en las escuelas, empoderarse de sus observaciones y generar una identidad de grupo que le retó a transformar la cultura escolar que estaba viviendo, fundamentalmente en relación con su imagen de infancia, de enseñanza y su rol docente.

Enfrentarse a este contexto nuevo, holístico, sistemático y cooperativo donde el eje, la mirada y el análisis se sitúan en los niños y niñas, ha provocado que el pensamiento práctico de María esté en todo momento alerta y en tensión, fundamentalmente en las fases de diagnóstico y diseño de la LE cuando inicia el movimiento de teorizar la práctica.

Un proceso que eclosiona en las fases de desarrollo y revisión de la LE, cuando María, al experimentar la teoría, crea, convive e incorpora las nuevas concepciones, actitudes, habilidades, emociones y valores en una estructura que hace suya y que le ayuda a desenvolverse con satisfacción en este nuevo ecosistema de aprendizaje transdisciplinar y de pluralidad metodológica.

Finalmente, cabe destacar la importancia de la tutoría que acompaña a María en sus inquietudes y que al mismo tiempo que estimula la identidad de grupo en un ambiente de confianza, la reta a generar una propuesta que trasgrede la cultura escolar que están viviendo en sus contextos de prácticas. Decisiones complicadas que la TA canaliza a través del fomento de hábitos reflexivos, donde el grupo es dueño y responsable la LE, haciendo visible sus decisiones y la tentación constante de repetir una cultura escolar caduca. Además, cabe destacar la relevancia de que la TA sea experta en LS permitiéndole organizar y guiar con destreza, creatividad y profesionalidad esta compleja LS.

El análisis de la información nos ha permitido evidenciar cómo María actúa, se emociona, reflexiona, debate, se equivoca o rectifica... en distintas circunstancias y procesos de aprendizaje diversos. María ha pasado de tener una actitud técnica a construir un rol docente observador y reflexivo. Y ha sabido deshacer la dicotomía teoría y práctica, para tejer de forma orgánica una experiencia de sentido y relación entre cada uno de los elementos que la constituyen: reflexión, experiencia, infancia y cooperación.

Esta investigación podría seguir ampliándose y profundizando en las fases de evaluación y revisión de la LE, que no ha podido ser observada; sería interesante profundizar en cómo las tablas de actuación docente y evaluación de los aprendizajes actúan en contextos de formación inicial, ya que en investigaciones en formación permanente destacan por su virtualidad pedagógica (Soto et al., 2016).

\section{Apoyos}

A los participantes en esta investigación, en especial a María que nos ayuda a seguir creciendo en la formación de futuros docentes. Esta investigación se desarrolla en el marco del Proyecto de I+D 2017: Lesson Studies, escuela y universidad: investigando la reconstrucción del conocimiento práctico en la formación inicial del profesorado (EDU 2017-86082-p), financiado por el Programa Estatal de Fomento de la Investigación Científica y Técnica de Excelencia, 
subprograma estatal de generación de conocimiento, y la ayuda prestada por el Ministerio de Ciencia, Innovación y Universidad con la beca de Formación del Profesorado Universitario.

\section{Referencias}

Alsina, A. y Batllori, R. (2015). Hacia una formación del profesorado basada en la integración entre la práctica y la teoría. Una experiencia en el Practicum desde el modelo realista. Investigación en la Escuela, 85, 5-18.

Argyris, C. (1993). Conocimiento para la acción. Granica.

Blakemore, S. y Frith, U. (2005). Cómo aprende nuestro cerebro. Las claves de la educación. Planeta.

Blanco, N. (1999) Aprender a ser profesor/a: el papel del practicum en la formación inicial. En A. Pérez Gómez, J. Barquín Ruiz y J. F. Angulo Rasco (Eds.), Desarrollo profesional del docente. Politica, investigación y práctica (pp. 398-379). Akal.

Borges, J. L. (2005). El libro de arena. Emece.

Bueno i Torrens, D. (2017). Neurociencia para educadores. Octaedro.

Chassels, C. \& Melville, W. (2009). Collaborative, Reflective and Iterative Japanese Lesson Study in an Initial Teacher Education Program: Benefits and Challenges. Canadian Journal of Education / Revue canadienne de l'éducation, 32, 734-763. https://doi.org/10.2307/canajeducrevucan.32.4.734

Cladinin, J. \& Husu, J. (2017). The Sage handbook of Research on teacher education. Sage.

Damasio, A. (2003). En busca de Spinoza. Neurobiología de la emoción y los sentimientos. Destino.

Darling-Hammond, L. \& Oakes, M. (2019). Preparing Teachers for Deeper Learning. Harvard Education Press..

Elliot, J. (1999). La relación entre comprender y desarrollar el pensaminto de los docentes. En A. Pérez Gómez, J. Barquín Ruiz y J. F. Angulo Rasco (Eds.), Desarrollo profesional del docente. Politica, investiación y práctica (pp. 364-378). Akal.

Elliot, J. (2015). Lesson y learning Study y la idea del docente como investigador. Revista Interuniversitaria de Formación del Profesorado, 84(29.3), 29-46.

Elliot, J. (2016). Significant themes in developing the theory and practice of lesson study. International Journal for Lesson and Learning Studies, 5(4), 274-280. https://doi.org/10.1108/IJLLS-08-2016-0022

Orden ECI/3854/2007, de 27 de diciembre, competencias Maestro en Educación Infantil. Boletín Oficial del Estado, 29 diciembre de 2007. Recuperada de https://www.boe.es/eli/es/o/2007/12/27/eci3854

Gallardo, M., Guillén, F., Mayorga, M. J. y Sepúlveda, P. (2020). Identificación de factores que afectan la satisfacción del alumnado de educación sobre la tutorización en su formación práctica. Un estudio con ANOVA. Formación Universitaria 13(3), 147-156.

Gil Flores, J. y Perera Rodríguez, V. H. (2001). Análisis informatizado de datos cualitativos: introducción al uso del programa NUD.IST-5. Kronos.

Grossman, P., Hammerness, K. \& McDonald, M. (2009). Redefining teacher: re-imagining teacher education. Teachers and Teaching: Theory and Practice, 15(2), 273-290.

Hagger, H. \& Hazel, H. (2006). Learning Teaching from Teachers: Realising the Potential of School-Based Teacher Education. Open University Press.

Khaneman, D. (2015). Pensar rápido, pensar despacio. Debate.

Korthagen, F. (2010). La práctica, la teoría y la persona en la formación del profesorado. Revista Interuniversitaria de Formación del Profesorado, 24(2), 83-101.

Lamb, P., Kathleen, L., \& Aldous, D. E (2012). Enhancong the spaces of reflection: A buddy peer-review process within physical education initial teacher education. European Physical Education Review, 19(1), 21 38.

Martin, D. \& Clerc-Georgy, A. (2015). Use of theoretical concepts in lesson study: an example from teacher training. International Journal for Lesson and Learning Studies, 4(3), 261-273. https://doi.org/10.1108/IJLLS-10-2014-0039

Norwich, B., Dudley, P. \& Ylonen, A. (2014). Using Lesson Study to assess pupils' learning difficulties. International Journal for Lesson and Learning Studies, 3 (2), 192-207. https://doi.org/10.1108/IJLLS12-2013-0059

Pérez Gómez, A. I. (1999). El practicum de enseñanza y la socialización profesional de los futuros docentes. En A. Pérez Gómez, J. Barquín Ruiz y J. F. Angulo Rasco (Eds.), Desarrollo profesional docente. Política, investigación y práctica (pp. 636-660). Akal.

Pérez Gómez, A.I (2012). Educarse en la era digital. Morata.

Pérez Gómez, A.I., Soto, E. y Serván, M.J. (2015). Lesson Study; re-pensar y re-crear el conocimiento práctico en cooperación. Revista Interuniversitaria del Profesorado, 84(29.3), 81-101.

Po Yuk, K. (2011). Critical conditions for pre-service teachers' learning through inquiry. International Journal for Lesson and Learning Studies, 1(1), 49-64. https://doi.org/10.1108/20468251211179704 
Prieto, A. y Giménez, X. (2020). La enseñanza universitaria basada en la actividad del estudiante: evidencias de su validez. En N. De-Alba-Fernández y R. Porlán (Eds.), Docentes universitarios. Una formación centrada en la práctica (pp. 23-55). Morata.

Punset, E. (2014). El mundo en tus manos. Destino.

Soto, E., Pérez Gómez, A.I \& Rodríguez, C. (2020) Aprender a enseñar en la universidad: de la investigación Acción a la Lesson Study. En N. De-Alba-Fernández y R. Porlán (Eds.), Docentes universitarios una formación centrada en la práctica (pp. 55-83). Morata.

Soto, E., Serván, M.J. \& Caparrós, R. (2016). Learning to teach with Lesson Study: The practicum and the degree essay as the scenario for reflective and cooperative creation. International Journal for Lesson and Learning Studies, 5(2), 116-129. https://doi.org/10.1108/IJLLS-12-2015-0042

Soto, E., Serván, M.J. Peña, N. \& Pérez Gómez (2019) Action research through lesson study for the reconstruction of teachers practical knowledge. A review of research at Málaga University (Spain), Educational Action Research, 27(4), 527-542. https://doi,org/10.1080/09650792.2019.1610020.

Xu, H. \& Pedder, D. (2015). Lesson Study: an international review of research in Teacher Education. In PDudley, Lesson Study. Professional learning for our time (pp. 29-59). Routledge.

Zeichner, K. (2010). Nuevas epistemologías en formación del profesorado. Repensando las conexiones entre las asignaturas del campus y las experiencias prácticas en la formación del profesorado en la universidad. Revista Interuniversitaria de Formación del Profesorado, 68(24.2), 123-151.

Zeichner, K. \& Gore, J. (1990). Teacher socialization. In W. Houston, (Ed.), Handbook of research on teacher education (pp. 329-348). McMillan. 